\title{
Development of a Conductometric Sensor to Approximately Estimate the Plasma Osmotic Pressure of Blood Using a Novel Glass-Bead-Based Blood Cell Filter
}

\author{
Shigeru Toyama ${ }^{1, *}$, Masanori Nakamura ${ }^{1,2}$, Ron Usami ${ }^{2}$ and Seishi Kato ${ }^{1}$ \\ ${ }^{1}$ Research Institute, National Rehabilitation Center for Persons with Disabilities, \\ 4-1 Namiki, Tokorozawa, Saitama 359-8555, Japan \\ ${ }^{2}$ Department of Applied Chemistry, Faculty of Engineering, Toyo University, \\ 2100 Nakanodai, Kujirai, Kawagoe, Saitama 350-0815, Japan
}

(Received October 19, 2009; accepted March 30, 2010)

Key words: conductometric sensor, blood cell filter, plasma osmotic pressure, dehydration

A conductometric sensor for approximately estimating the plasma osmotic pressure of blood has been developed. The sensor was designed to spontaneously absorb blood upon contact, then separate plasma by adsorbing blood cells, and finally measure the conductance of the plasma using built-in electrodes. This sensor used a blood cell filter made up of a number of glass beads. The diameter of the glass beads was $0.063-$ $0.088 \mathrm{~mm}$, which was one order of magnitude larger than that of blood cells, so that the adsorbed blood cells on the beads did not prevent further blood flow through the space between the beads. We used preserved horse blood to evaluate this sensor. The filter successfully absorbed test blood and provided plasma to the measurement element in the sensor. An alternating voltage $(10 \mathrm{mV}(\mathrm{pp}), 1.5 \mathrm{kHz})$ was applied between the builtin platinum electrodes and the corresponding current was measured simultaneously to calculate the conductance of the plasma. The sensor output was independent of hematocrit from 20 to $40 \%$ and exhibited a linear relation to plasma osmotic pressure from 360 to $460 \mathrm{mOsm} / \mathrm{L}$. The osmotic pressure was artificially controlled by $\mathrm{NaCl}$.

\section{Introduction}

Dehydration is an abnormal physical state in which the amount of body fluid decreases extremely, often accompanying an increase in electrolyte concentration. ${ }^{(1)}$ It sometimes happens to aged people, infants, and postoperation patients. Dehydration often leads to headache, vertigo, and unconsciousness, and is lethal in the worst case. In particular, it is hazardous for those who cannot convey their physical condition to other people, such as infants or unconscious patients. It is very important to take up appropriate amounts of water with appropriate electrolytes to prevent such serious

${ }^{*}$ Corresponding author: e-mail address: toyama-shigeru@rehab.go.jp 
situations. The osmotic pressure of blood plasma provides indispensable information to make a prompt and accurate diagnosis of dehydration for the appropriate treatment of patients.

A popular method of measuring plasma osmotic pressure is to use an osmometer, which is a cryoscopic measurement system. In this case, we have to separate plasma from blood by centrifugation before using the osmometer. Another conventional method is to estimate osmotic pressure from the concentration of the major components of blood such as major ions, urea nitrogen, and glucose. These conventional methods require us to sample blood from patients with a collection syringe, which causes several problems. The whole process consumes at least $30 \mathrm{~min}$, which is insufficient for the prompt diagnosis of acute abnormalities. Moreover, these methods require professional blood sampling techniques and a medical license in most countries.

To meet the requirement of prompt diagnosis, we have been developing an osmotic pressure sensor. Our final goal is to develop a portable sensor using a disposable sensor chip that can be changed after every measurement similarly to a commercial glucose sensor. Our first trial for achieving this purpose was to fabricate a multisensor system that measures the concentrations of major components of blood and estimates osmotic pressure from the measured outputs. ${ }^{(2)}$ However, it costs too much to fabricate these sensors with reproducible performance on a small chip.

Another method of estimating plasma osmotic pressure is to fabricate a miniaturized Coulter Counter using micro-electromechanical systems (MEMS) technology.(3,4) The drawback of this system is that it requires flow pumping mechanisms. A sensor without any mechanical parts is advantageous.

We adopt here conductometry, a simple way to roughly determine the osmotic pressure. In general, the conductance of a bulk liquid correlates with the ionic concentration and mobility in solution. Conductometry seems useful for roughly estimating osmotic pressure because the concentrations of ionic species in plasma primarily contribute to osmotic pressure. The drawback is that conductometry alone cannot detect dehydration in some special cases, such as dehydration due to hyperglycemia, because conductometry does not reflect changes in the concentration of neutrally charged components of blood. However, dehydration can be detected by other measurement tests, e.g., glucose sensing, in such a case.

To develop a sensor, we need a separation filter for blood cells in conductometry because the movement of ions is impeded by the existence of blood cells. A conventional way to block blood cells from contacting the sensor surface is to place a membrane filter on the electrodes. ${ }^{(5)}$ Unfortunately, small changes in membrane posting on the electrode surface, i.e., posting position, formation of a void layer between the membrane and the electrode, and the degree of membrane compression, affect the electric field surrounding the electrodes, resulting in a marked change in the conductance. Although miniaturized cell filters have been reported, e.g., ref. 6, they are insufficient for our purpose because they require active flow systems. A smart sensor using three electrodes covered with a semipermeable membrane has been reported by Varlan et al. ${ }^{(7)}$ This sensor simultaneously estimates both hematocrit and plasma conductivity with a sophisticated calculation using the conductometric data obtained by these three electrodes. This 
sensor does not have any mechanical parts. However, the drawback of this sensor is that its measurement reproducibility depends on the structural controllability and fabrication reproducibility of the membrane. It seems to be difficult to attain these properties. Our approach here is to provide a blood cell filter that spontaneously absorbs blood and separates cells without requiring any mechanical parts.

\section{Experimental}

In some experiments, we used an electrode chip where a pair of electrodes was fabricated using conventional photolithographic technology. The substrate of the chip was a glass plate and the electrodes were platinum thin films. The area of each electrode was $1.5 \times 1.5 \mathrm{~mm}^{2}$, and the distance between the two electrodes was $0.9 \mathrm{~mm}$. The conducting pattern from the electrode to the terminal was covered with another glass plate or photoresist membrane.

To fabricate the blood cell filter, we used glass beads. Glass beads with a diameter of about $0.005 \mathrm{~mm}$ were obtained from Potters-Ballotini Co., Ltd., and those with diameters of $0.037-0.063 \mathrm{~mm}$ (No. 0.05), 0.063-0.088 mm (No. 0.07), and 0.105-0.125 mm (No. 0.10 ) were purchased from Toshinriko Co., Ltd. The glass beads were immersed in $5 \%$ nitric acid for $30 \mathrm{~min}$, washed with pure water, and then dried in an oven $\left(100^{\circ} \mathrm{C}, 2 \mathrm{~h}\right)$. Polylysine-coated glass beads were prepared by immersing the glass beads in a $0.1 \%$ polylysine solution (poly-L-lysine hydrobromide, Nacalai Tesque, Inc., MW > 300,000) for $20 \mathrm{~min}$, washing them with pure water, and then drying them in an oven $\left(80^{\circ} \mathrm{C}, 2\right.$ h). The glass beads were packed into a glass tube (typical diameter was $4.0 \mathrm{~mm}$ ) and the end of the glass tube was plugged with cotton. The final conductometric sensor was fabricated by inserting two electrodes into the filter. The size of each material of the filter and the sensor is explained in the figure captions.

Preserved horse blood ( $\mathrm{pH}$ 7.4) was purchased from Nippon Biotest Laboratories Inc. The purchased blood contained Alsever's solution at half the final volume to maintain storage stability, and we used it within about two weeks after purchase. Sample blood with the desired hematocrit was reconstituted by mixing plasma and blood cells, which were separated from the preserved horse blood by centrifugation beforehand. The serum samples were regenerated by adding water to a lyophilized human serum (Control Serum I, Wako Pure Chemical Industries, Ltd.). A serum sample with the desired concentration was prepared by changing the water/lyophilized serum ratio.

The conductance between the electrodes was determined using a chemical impedance analyzer (CSYS98 System, Tohogiken Co., Ltd.). In a typical case, a sinusoidal wave $(10 \mathrm{mV}(\mathrm{pp}), 1.5 \mathrm{kHz})$ was applied between the electrodes and the resultant current was simultaneously measured to calculate conductance (i.e., the real part of $\mathrm{I} / \mathrm{V}$, where $\mathrm{I}$ and $\mathrm{V}$ are the amplitudes of the current and applied voltage, respectively). In the case of experiments using the electrode chip, the terminals of the electrodes were connected to the impedance analyzer and dipped into the bulk sample solution, and then conductometric measurement was carried out. In the case of the measurement with the filter-incorporated sensor, the tip of the sensor was dipped into the sample solution. After a spontaneous absorption of sample solution into the sensor, the conductometric 
measurement was carried out. The estimation of the osmotic pressure of the sample liquids was carried out using Osmotron-5 (Orionriken Co., Ltd.), a cryoscopic measurement apparatus.

\section{Results and Discussion}

In preliminary experiments, we found that the current between the electrodes was not stable when a constant voltage was applied. The application of the constant voltage to an electrode might cause electrochemical oxidation/reduction at the electrode surface, and change the surface potential of both electrodes, leading to a gradual change in the current. Therefore, we applied an alternating voltage between the electrodes (Fig. 1(a)). The

(a)

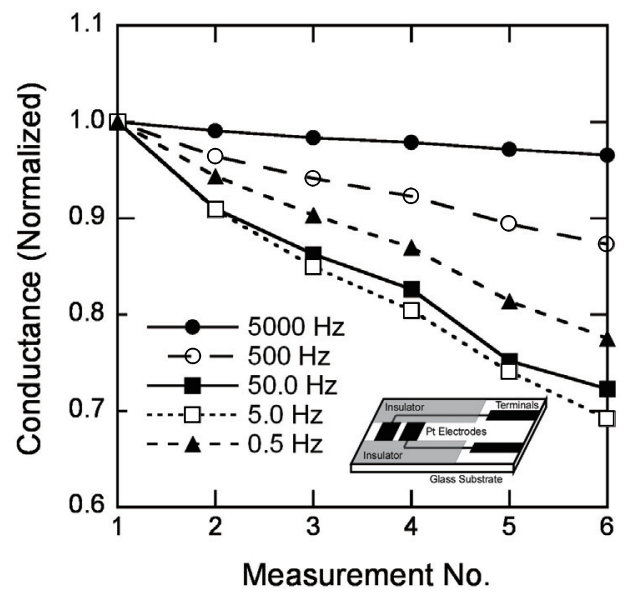

(b)

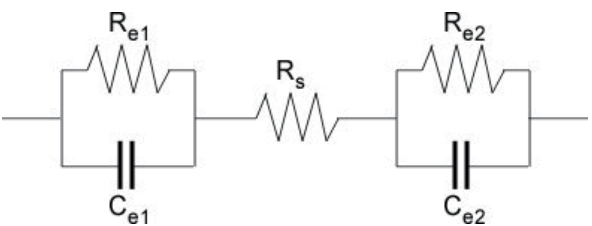

Fig. 1. Conductometric measurement using a pair of electrodes. (a) Stability dependence of conductance on frequency. The sample solution was $150 \mathrm{mM} \mathrm{NaCl}$. An alternative voltage (10 $\mathrm{mV}(\mathrm{pp})$ ) was applied between electrodes. The inset shows the general structure of the electrode chip used in this experiment. The chip was inserted into a bulk sample solution and conductometry was carried out. The measurement was performed repeatedly. In each measurement cycle, current/ voltage was measured with various frequencies. The measurement number in the figure represents the number of measurement cycles. (b) Equivalent circuit corresponding to the measurement environment including electrodes. $E_{1}$ and $E_{2}$ represent the two electrodes. $R_{\mathrm{e} 1}$ and $R_{\mathrm{e} 2}$ represent the resistance at the surface of electrodes. $C_{\mathrm{e} 1}$ and $C_{\mathrm{e} 2}$ represent the capacitance of the electric double layer at the surface of electrodes. $R_{\mathrm{s}}$ represents the bulk resistance of the sample solution. 
stability of the current was improved when the frequency was increased. This result can be explained with a diagram of equivalent circuit corresponding to the electrode system (Fig. 1(b)). The current through the electrode was attributed to both the electron transfer to/from the electrochemically active species in the solution and the capacitive current due to the formation of an electric double layer at the electrode surface. In the case of the constant voltage, the contribution of the capacitive current was zero; it increased when frequency increased in the case of applying an alternating voltage. Furthermore, we obtained a linear relation between the conductance and the concentration of the $\mathrm{NaCl}$ solution as previously expected (data not shown). Therefore, we used the alternating voltage in further experiments.

The results of the conductometric measurement of regenerated serum are shown in Fig. 2. Both conductance and osmotic pressure exhibited a good linear relation to the concentration of plasma, suggesting that conductance is a good indicator of osmotic pressure. Therefore, conductometric measurement can be used to estimate osmotic pressure as long as the sample solution is serum.

However, it is difficult to apply conductometry to the analysis of whole blood because whole blood contains blood cells such as erythrocytes. Figure 3 shows the dependence of conductance on hematocrit (i.e., the cell volume in blood). Conductance decreased with an increase in hematocrit. This result can be explained by considering ionic mobility. Generally, the total current through the electrolyte depends on the numbers of ions, distance between electrodes, sectional area of the ionic pathway, and mobility of ionic species. In the case of blood, blood cells behave as obstacles for ionic movement.

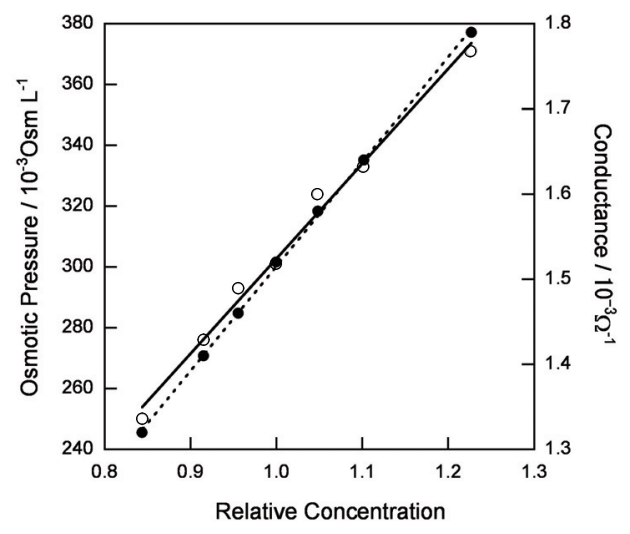

Fig. 2. Conductance $\left(\bullet:\right.$ dotted line; $\left.Y=1.23 X+0.288, r^{2}=0.999\right)$ calculated from the results of impedance measurement, and osmotic pressure ( $\circ$ : solid line; $Y=312 X-9.41, r^{2}=0.991$ ) measured by an osmometer as a function of the concentration of reconstituted serum. To estimate conductance, an alternative voltage $(1.5 \mathrm{kHz}, 10 \mathrm{mV}(\mathrm{pp}))$ was applied between electrodes. The horizontal axis represents the relative concentration normalized by the recommended concentration to regenerate normal serum. The electrode chip for this experiment was the same as that of Fig. 1. 


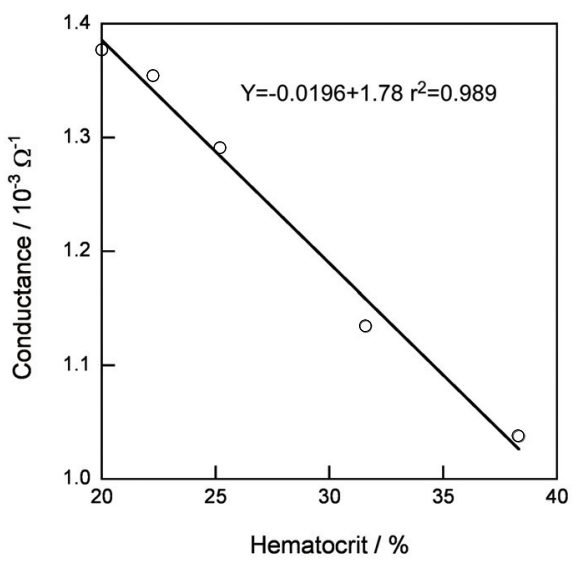

Fig. 3. Conductance as a function of the hematocrit of reconstituted horse blood. In this experiment, only the hematocrit was changed, while the concentration of plasma remained constant. The electrode chip for this experiment was the same as that of Fig. 1.

The existence of blood cells increases the effective length between electrodes, and decreases the effective sectional area of the ionic pathway, decreasing ionic conductivity.

One problem that arises here is that the direct measurement of the conductance of whole blood is insufficient to estimate the level of dehydration. Unfortunately, dehydration causes increases in both the concentration of blood plasma and hematocrit. The former increases the conductivity, while the latter decreases it at the same time. Therefore, it is complicated to interpret the conductivity of whole blood. To determine the dehydration stage, we have to know the normal hematocrit. However, hematocrit varies from person to person. Moreover, it depends on a person's condition.

To avoid interference in hematocrit measurement in conductometry, a promising solution is to eliminate blood cells before the measurement. Although the simplest way to obtain plasma from whole blood is centrifugation, this is not suitable for our purpose. Another way is to use a blood cell filter to separate plasma from the whole blood sample. We used glass beads as a filtering material, which were packed in a tube. Such a filter structure enables the sample solution to be spontaneously absorbed by capillary action, so that the sensor does not require any flow pumps.

To elucidate the general characteristics of the glass-bead-based filter, we performed several preliminary experiments. Figure 4 shows a plot of the absorption speeds of water through glass-bead-filled tubes. There was an optimum size of glass beads to maximize absorption speed (Fig. 4(a)). The water absorption speed was largest when the diameter of the glass beads was between 0.036 and $0.088 \mathrm{~mm}$. The reason for the existence of the optimum diameter is not exactly clear at present, but several factors may influence absorption speed: the gravity in the sample liquid, the hydrophilicity at the surface of glass beads, the viscosity of the sample liquid, and the pass length of water in the lattice of the beads. Another factor, i.e., the surface effect of the glass tube, was experimentally 
(a)
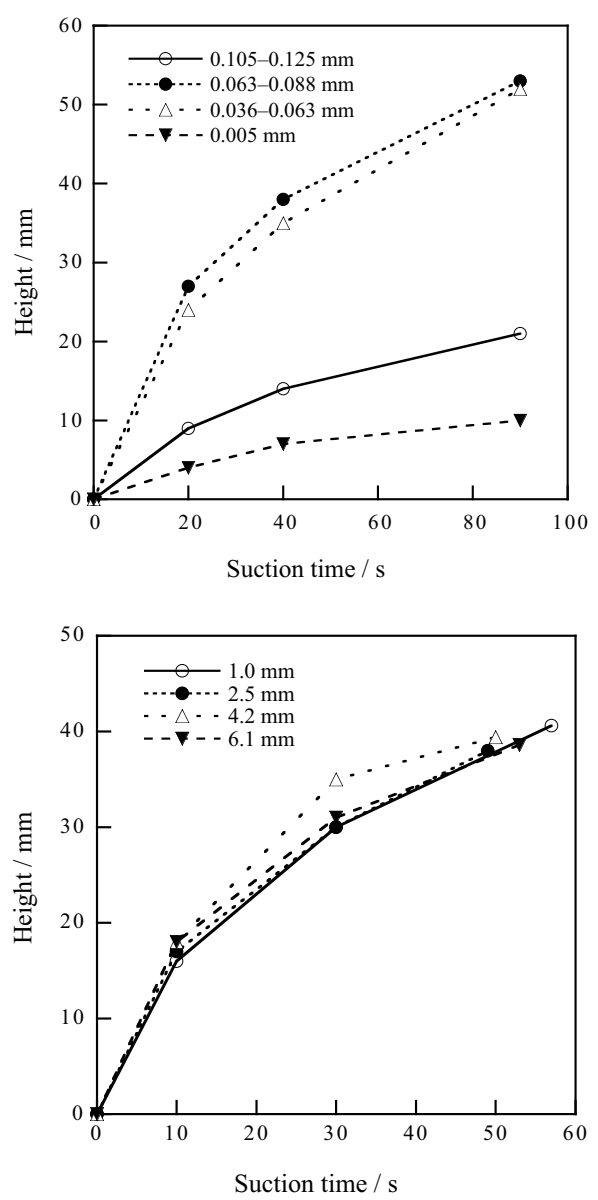

Fig. 4. Absorption of water into bare-glass-bead-filled glass tubes. (a) Dependence of absorption height on the diameter (the values described in the graph) of glass beads. The inner diameter of the glass tube was $4.0 \mathrm{~mm}$. (b) Dependence of absorption height on the inner diameter (the values described in the graph) of glass tubes. The diameters of the glass beads were $0.063-0.088 \mathrm{~mm}$.

excluded (Fig. 4(b)). If the glass beads are small, the effective area of the glass surface is large and the absorption power by capillary action seems dominant over gravity. However, the effective pathway for the sample liquid in the bead lattice becomes longer, decreasing absorption speed. Absorption speed also decreases when the diameter of the beads increases owing to the low absorption power.

In the case of whole blood, more complicated results were obtained. When bare glass beads of $0.005 \mathrm{~mm}$ diameter were used, the filter separated plasma at an extremely low absorption speed $(30 \mathrm{~mm}$ after $1 \mathrm{~h}$ ). On the other hand, the filter could not separate plasma when the diameter of the glass beads was $0.037-0.063 \mathrm{~mm}$. Because the average 
size of horse erythrocytes is about 5-6 $\mu \mathrm{m}$, the size selection effect may be dominant in those cases. Therefore, other mechanisms must be introduced to smoothly separate blood cells and plasma from whole blood at a high throughput. One possible way is to adsorb erythrocytes on the surface of glass beads.

We successfully fabricated a separation filter when we pretreated the glass beads with polylysine. Erythrocytes were clearly separated within an acceptable amount of time (Fig. 5). It took $10 \mathrm{~min}$ to fully adsorb blood solution, i.e., $50 \mathrm{~mm}$ (note that the absorption is $40 \mathrm{~mm}$ in the photograph). Since the surface of erythrocytes has negative charges, the cells are possibly captured by the positively charged polylysine coating on the surface of the glass beads. Although we pretreated the glass beads by simply immersing them in polylysine solution and carried out no further chemical immobilization, the filter apparently adsorbed blood cells. The marked change in the absorption property of the glass beads suggests that polylysine coated the surface and remained there even after the wash-out process. In our past experiments using surface plasmon resonance measurement, ${ }^{(8)}$ we observed that polylysine was tightly captured on a bare gold surface. We think that polylysine with its many positive charges binds to various substrates via electrostatic interaction. Polylysine is speculated to bind to the glass surface via the attractive force between the hydroxyl group of the glass surface and the amino group of polylysine.

Moreover, the filter was not clogged by the adsorbed blood cells. The reason for this may be the large difference in size between the glass beads and the horse blood cells. The diameter of the glass beads $(0.037-0.063 \mathrm{~mm})$ was one order of magnitude larger than that of horse erythrocytes (about $0.005 \mathrm{~mm}$ ).

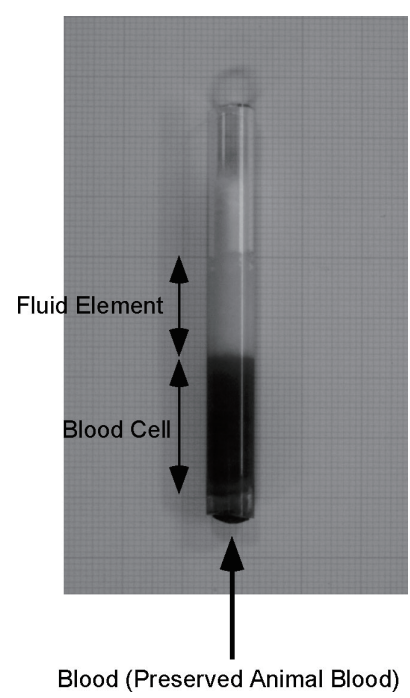

Fig. 5. Blood cell filter fabricated with the polylysine-pretreated glass beads (average diameter: $0.037-0.063 \mathrm{~mm}$ ), which were packed in a cotton-plugged glass tube (inside diameter: $5.8 \mathrm{~mm}$, length: $60 \mathrm{~mm}$ ). The length of the segment of the packed glass beads was $50 \mathrm{~mm}$. 
From the photograph in Fig. 5, the length of the red part $(24 \mathrm{~mm})$ was roughly estimated to be about $60 \%$ of that of the sample-absorbed part $(40 \mathrm{~mm})$. From these values, we can estimate the surface coverage of the blood cells adsorbed on the glass beads by the following calculation. The volume of the red part is calculated to be 634 $\mathrm{mm}^{3}$. If we assume a hexagonal close-packed structure of the glass beads, the partial volume of the glass beads is about $74 \%$ of the total volume. Then, the volume shared by the glass beads corresponding to the red part is calculated to be $469 \mathrm{~mm}^{3}$, which contains 7.16 $\times 10^{6}$ beads (assuming that the size of the beads is uniform at $0.05 \mathrm{~mm}$ ). Therefore, the total surface area of the glass beads corresponding to the red part is $2.81 \times 104 \mathrm{~mm}^{2}$. The volume of the whole blood sample is calculated to be $275 \mathrm{~mm}^{3}$, and the total volume of the blood cells is calculated to be $55 \mathrm{~mm}^{3}$ by considering the hematocrit of the preserved horse blood (about 20\%). We can obtain $1.96 \times 10^{3} \mathrm{~mm}$ as the average thickness of the adsorption layer on the glass beads surface. The calculated thickness is about $40 \%$ of the diameter of horse erythrocytes. Such low coverage may be attributed to the narrow space between two adjacent beads, through which cells are unable to pass. Therefore, we used larger beads $(0.063-0.088 \mathrm{~mm})$ to minimize such narrow spaces in the following experiments.

When two electrodes were inserted into the glass-bead-packed filter, conductance was almost independent of hematocrit from 20 to 40\%, as shown in Fig. 6. Furthermore, we obtained a linear relation between conductance and the osmotic pressure (from 360 to $460 \mathrm{mOsm} / \mathrm{L}$ ) of preserved horse blood when the osmotic pressure was artificially controlled with $\mathrm{NaCl}$ (Fig. 7). In this electrode configuration, the glass beads between the

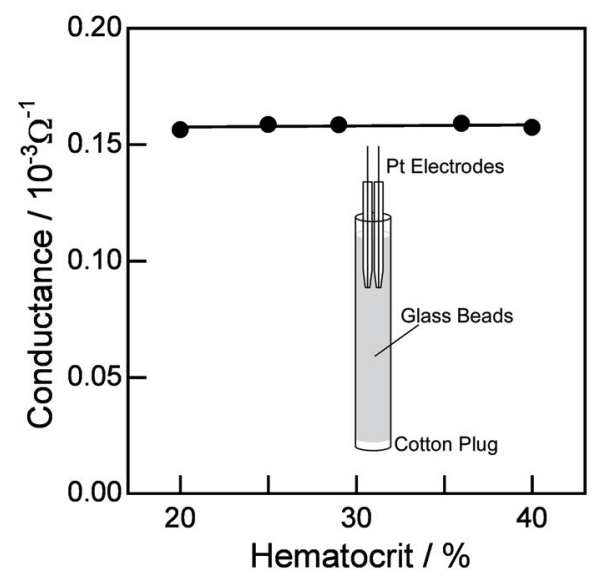

Fig. 6. Currents flow through the electrodes in a glass-bead-based filter as a function of hematocrit. The filter was fabricated with the polylysine-pretreated glass beads (average diameter: 0.063-0.088 $\mathrm{mm}$ ), which were packed in a cotton-plugged glass tube (inside diameter: $4.0 \mathrm{~mm}$, length: $60 \mathrm{~mm}$ ). The length of the segment of the packed glass beads was $50 \mathrm{~mm}$. Two platinum wire (diameter: $0.2 \mathrm{~mm}$ ) electrodes, which were encapsulated in glass capillaries (outer diameter: 1.6 $\mathrm{mm})$, were inserted into the glass tube (inserted length: $10 \mathrm{~mm})$. An alternative voltage $(1.5 \mathrm{kHz}$, $10 \mathrm{mV}(\mathrm{pp}))$ was applied between the electrodes. 


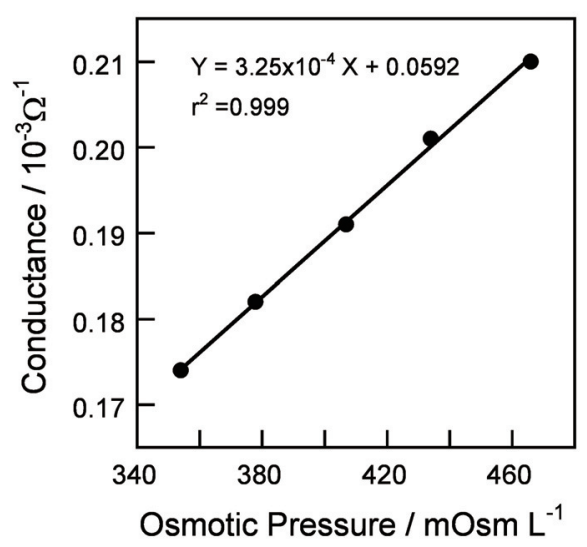

Fig. 7. Currents flow through the electrodes in a glass-bead-based filter as a function of the osmotic pressure of the preserved horse blood. The osmotic pressure was adjusted by adding concentrated $\mathrm{NaCl}$ solution $(1.0 \mathrm{M})$. An alternative voltage $(1.5 \mathrm{kHz}, 10 \mathrm{mV}(\mathrm{pp}))$ was applied between the electrodes. The osmotic pressure indicated here was obtained by measuring that of the plasma fraction after the centrifugation of blood.

electrodes lower ionic conductivity but do not affect the reproducibility of conductance owing to the packing reproducibility of the beads. The concentration of ionic species in the plasma only changes conductivity. Thus, we constructed a hematocrit-independent conductometric sensor. The total volume of the blood sample absorbed is calculated to be about $163 \mathrm{~mm}^{3}$, which is one order of magnitude larger than that of commercial blood glucose sensors. If we can insert narrower electrodes into the capillary tube, it should be easy to reduce the size of the sensor because the influence of the surface effect of the tube is small, as already explained above using the results in Fig. 4(b).

\section{Conclusion}

In this report, we proposed a conductometric sensor for estimating the osmotic pressure of whole blood. The most important part of this sensor is the glass-bead-based filter. By covering the surface of the glass beads with polylysine, we could use larger glass beads to increase sample absorption speed. The sensor composed of this filter and the electrodes successfully estimates the osmotic pressure of whole blood without any effect of hematocrit. The greatest advantage of this sensor is that it has no mechanical parts, such as a suction pump, to functionalize the cell filter. The sensor automatically absorbs samples, separates blood cells, and measures osmotic pressure by simply being brought into contact with the blood sample. Although further refinement is required, such as the simplification of the fabrication process of this sensor, we expect this sensor to be very useful for the rapid and accurate detection of dehydration in the future similarly to the personal blood glucose sensor used by diabetic patients. 


\section{References}

1 Maxwell and Kleeman's Clinical Disorders of Fluid and Electrolyte Metabolism, 5th ed., R. G. G. Narnis, C. R. Kleeman and R. G. Narins (eds.) (McGraw-Hill Inc., New York, 1994).

2 Y. Ikariyama, O. Takei, S. Yamauchi and S. Toyama: Dehydration Sensor: Digest of Technical Papers, The 10th International Conference on Solid-State Sensors and Actuators (Transducers '99) (1999) p. 1340.

3 M. Koch, A. G. R. Evans and A. Brunnschweiler: J. Micromech. Microeng. 9 (1999) 159.

4 R. Tanabe, S. Hata and A. Shimokohbe: IEEJ Trans. Sens. Micromachi. 126 (2006) 297.

5 J. Wang: Chem. Rev. 108 (2008) 814.

6 J. P. Brody, T. D. Osborn, F. K. Forster and P. Yager: Sens. Actuators, A 54 (1996) 704.

7 A. R. Varlan, P. Jacobs and W. Sansen: Sens. Actuators, B 34 (1996) 258.

8 S. Toyama, K. Aoki and S. Kato: Sens. Actuators, B 108 (2005) 903. 